For Internal Distribution Only

\author{
Experimental Support and Facilities Division \\ Collider-Accelerator Department \\ BROOKHAVEN NATIONAL LABORATORY \\ Upton, New York 11973
}

ES\&F Division Technical Note \# 165

\title{
Development of the Pixel Chamber
}

I-Hung Chiang, Adam Rusek, Daniel Ottavio, Dysart Ravenhall, Steve Bellavia

July 15, 2009 


\section{Development of the Pixel Chamber}

I-Hung Chiang, Adam Rusek, Daniel Ottavio, Dysart Ravenhall and Steve Bellavia

I. Historical Background.

The ionization chamber has been used for centuries recording the radiation. In the early 70 , the wire chamber becomes an important device for particle track detection. In the accelerator application, the segment wire ionization chamber becomes a useful beam monitor device. In the early 80 , it was realized in the AGS experimental area that with added integration electronics, the application of SWIC, (Segmented Wire Ionization Chamber) could be extended to the lower intensity beam. It was decided to apply this technique to the SWIC, using small diameter wire to provide gain and integrated electronics to integrate the low level signal. This device was then used in delivery of the Heavy Ion beam, typically $10 * * 5$ or less fully stripped Gold, to the experimental area. It was also apply to even lower level of beam, $10^{\wedge} 6-10^{\wedge} 7$ single charged particles, in the secondary beam line.

II. Application of SWIC in the beam line:

In the newly built beam line, we instrumented it with a SWIC and Ion Chamber. These devices were used for beam size and intensity monitor. It provides the signal to display the beam in the oscilloscope. It had been indispensible in beam tuning. Fig. 1 shows the profile display of the beam. 

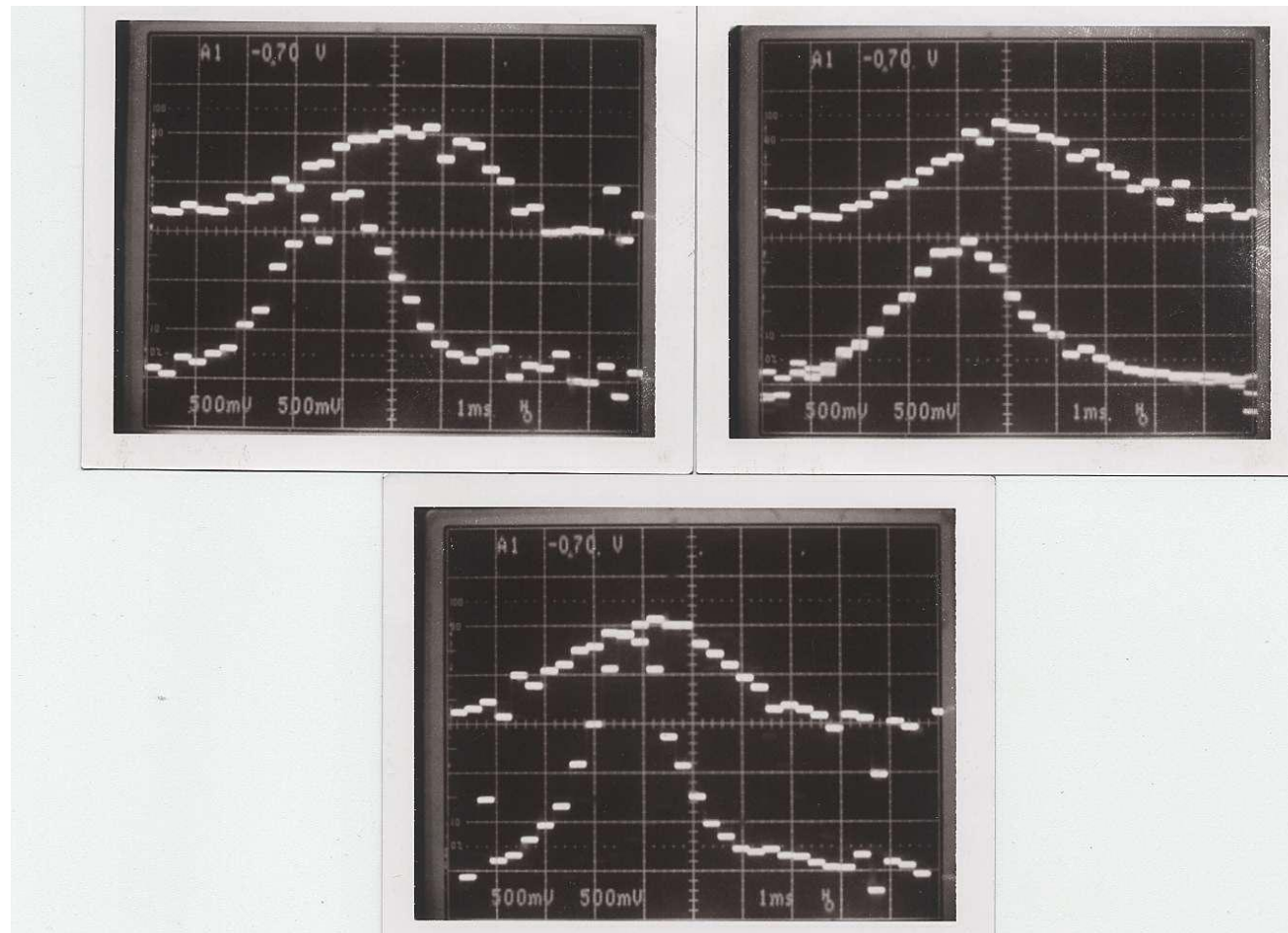

Fig. 1 Scope display of SWIC signal.

The distribution shows 32 wires in each plane, horizontal and vertical. This way, beam tuning could quickly see the beam size and location of the beam. It was a very intuitive display for quick response. Since the number of wires is limited to 32 per plane, the wire spacing is adjusted according to the beam size. The electronics was designed to be very simple so that it could operate stand alone in the field without much attention. The display was done with oscilloscope. After computers became more abundant, it was then interfaced with our control system with software to plot the profile. The integrator was designed with Op-amp. Fig. 2 shows the basic diagram. It has two different capacitors, 1 nano-farad and 9 nano-farad. This way, the dynamic range could be changed by factor of 10. In the later version, the lower range was moved down to $470 \mathrm{pf}$, and $300 \mathrm{pf}$. 
There is an inverting switch which is used to inverting the output signal. This way, we could operate the chamber in different bias voltage polarity.

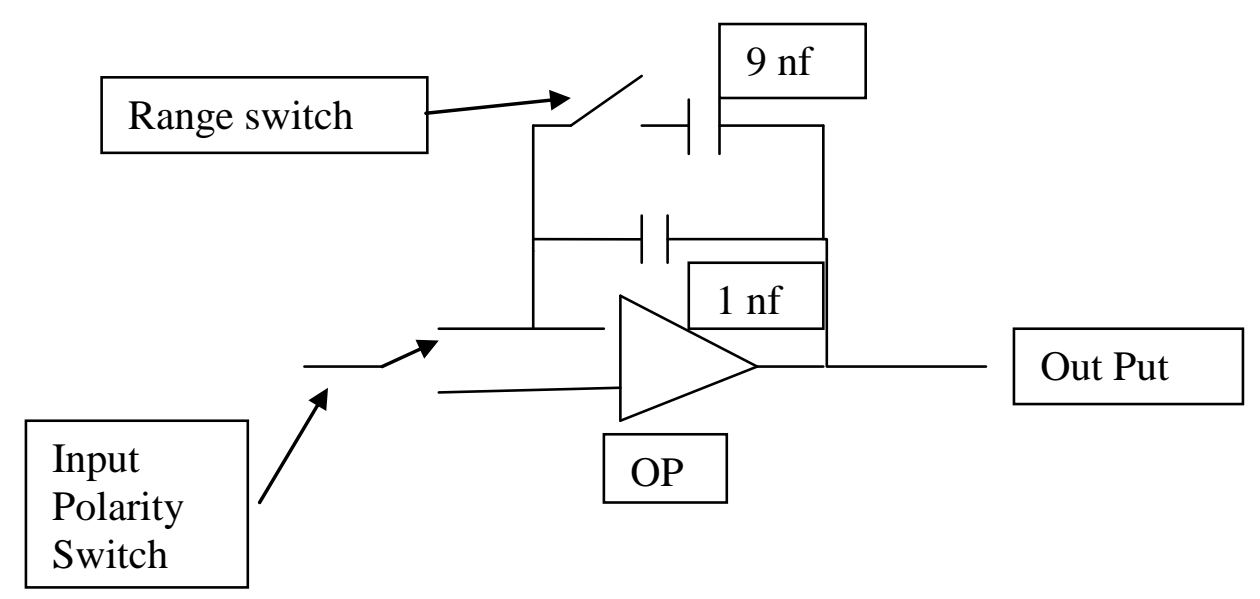

Fig. 2 Sketch of the Integrator circuit.

III. Motivation of making Pixel Chamber.

Before the completion of the NSRL facility, there was a plan to expand the capability of the beam line to lower beam intensity. The most obvious way to do that was to use Scintillators for Dosimetry and beam monitoring. The technique is very well established and we had enough expertise in house to carry out this instrument. The only question is how fine we will need to segment the device. The more we segmented it the more costly and complex the system became. The idea led to trying out the pixel chamber with gain. It is well known that this technique was used in Particle Physics to detect charge particles. It concern mostly with leading edge of the fast signal. The question is whether it will truly reflect the intensity of the beam distribution. We want to integrate the total signal. When there is no gain, the electron signals reach the collecting plate within a few hundred nanoseconds while the positive ion takes on the order of a millisecond. With large gain, half of signal will arrive at the collecting plate in a stream last about millisecond, after initial spike. It is also questionable whether 
our existing electronics will be capable of integrating these signals.

IV. Initial try:

In our standard SWIC package, we could combine wire and bias plane in a different way, Fig 3. shows the air SWIC assembly. Each wire plane, Horizontal and Vertical, are sandwich in two Bias Planes.

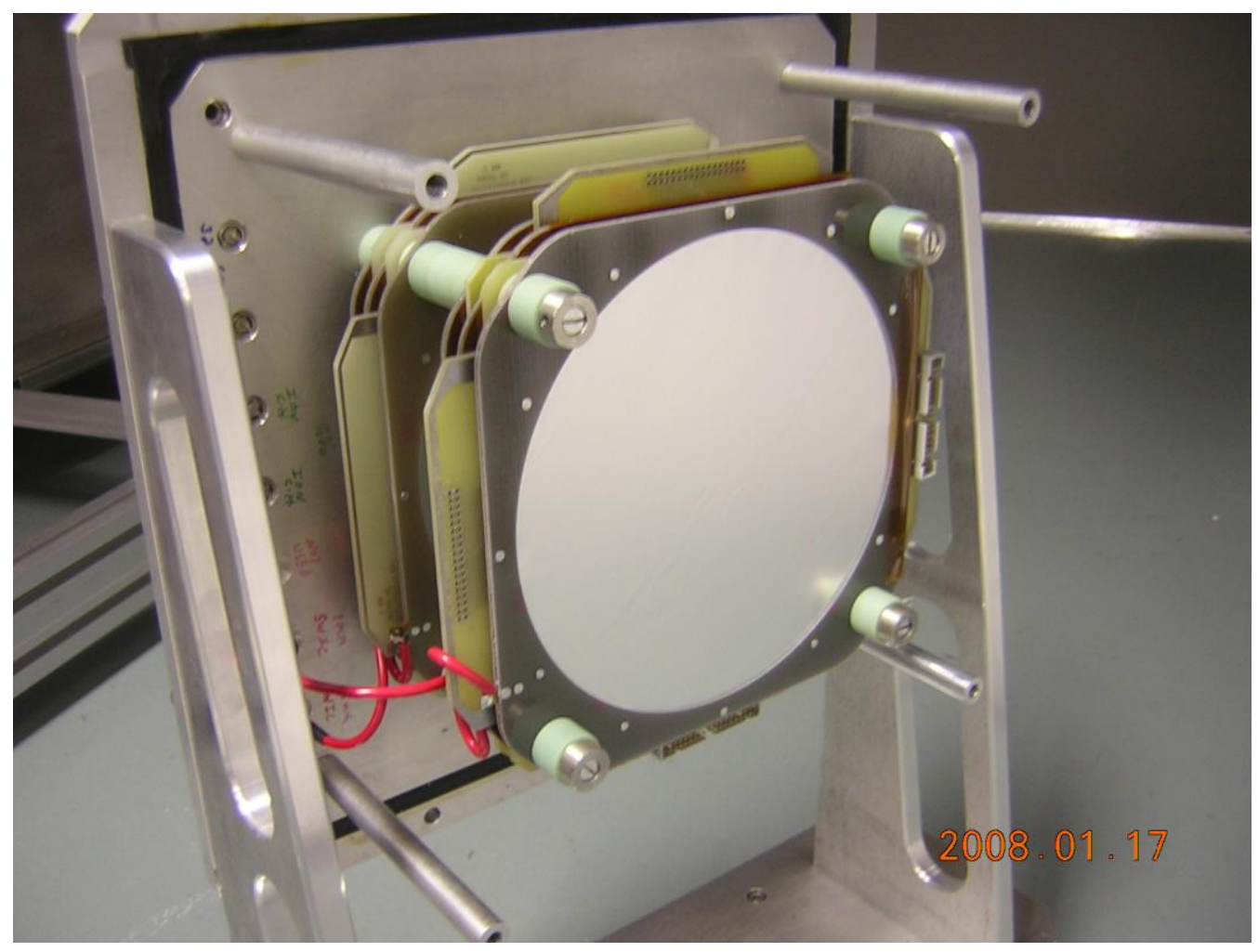

Fig. 3 Air SWIC assembly.

We decided to modify the chamber to act as a pixel chamber. The wire plane was tied to + high voltage and we made a pixel plan with FR-4 board. Fig. 4 shows the pixel plane, which is at "ground" potential. 


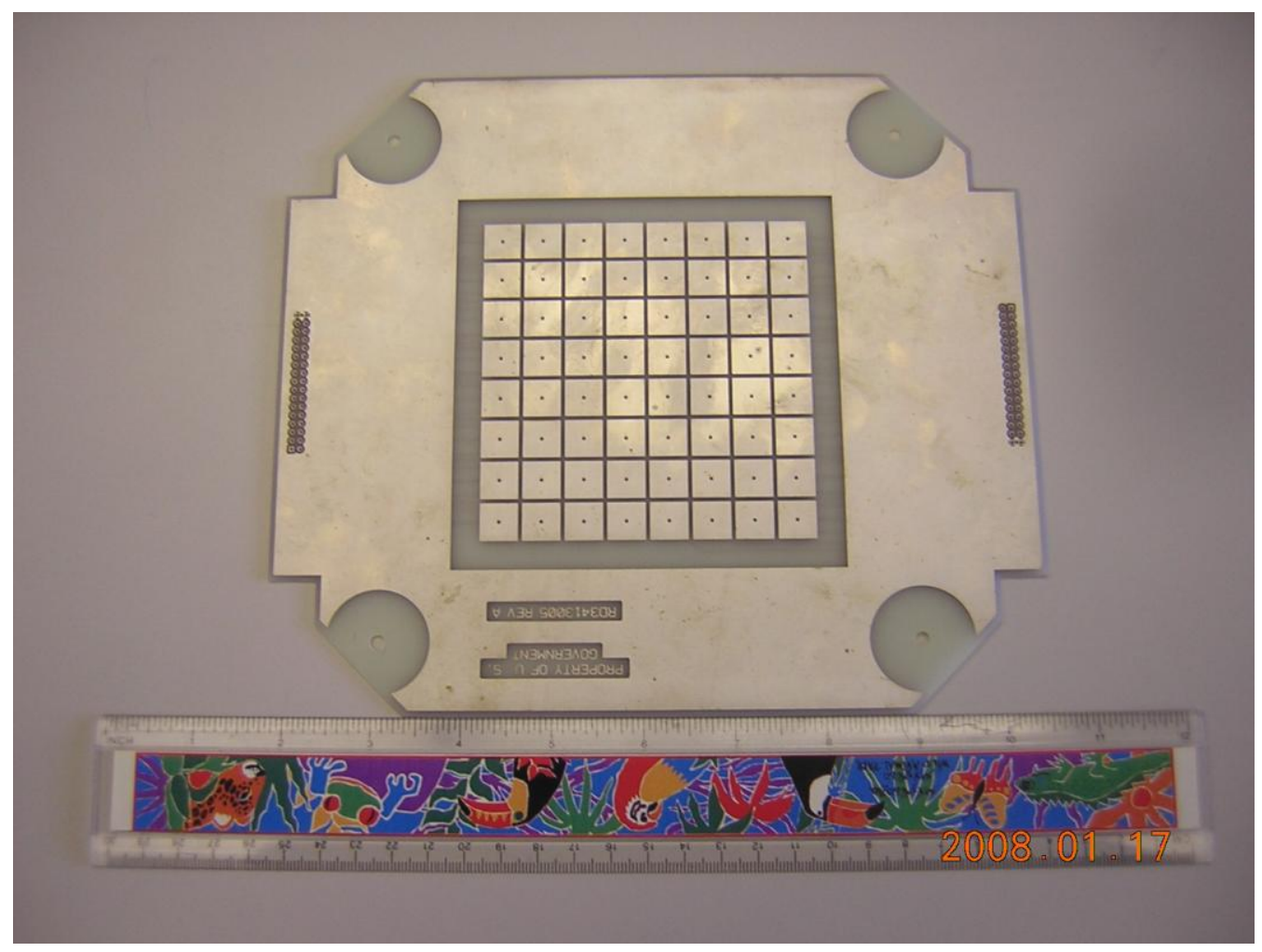

Fig. 4. Small test Pixel Plane

The pixel size was chosen to be $1.2 \mathrm{~cm} \times 1.2 \mathrm{~cm}$, because the wire spacing of the wire chamber is $3 \mathrm{~mm}$. This way, all Pixels will face 4 wires. The chamber size is only 12 $\mathrm{cm} \times 12 \mathrm{~cm}$, which limits the choice of pixel size. We supply the + high voltage on the wire plane. This way, the electron drifts to the wire. We use 0.7 mil diameter gold plated Tungsten wire for the Anode. The electrical field near the wire is proportional to $1 / \mathrm{r}$. The wire spacing is 3 $\mathrm{mm}$ and distance from the wire plan to pixel plan is $3 / 16$ inches. The capacitance of this design is $5.27 \mathrm{pf} / \mathrm{meter}$. At 1000 volts, the electrical field at the surface of the wire is $105,000 \mathrm{volts} / \mathrm{cm}$. At this field, the electrons may get enough energy to start ionizing the molecules it collides with. The collision length is of the order of 0.5 micron. That is the principle of gain in wire chambers. The gain grows with high voltage bias exponentially. The ionization 
potential of Argon is $15.7 \mathrm{eV}$. For 0.5 micron collision length, it needs a field of 300,000 volts $/ \mathrm{cm}$ for electron to gain enough energy to ionize the Argon gas in one collision length. At 1000 volts setting, it will require electron to miss colliding with gas for a few collision lengths. Even at very high bias voltage, the $1 / \mathrm{r}$ effect will reduce the field after a few wire diameters such that there is no multiplication at all.

The initial try shows encouraging results. There indeed was gain and we could see signal. Unfortunately, the beam is larger than our detector. The beam is larger than $20 \mathrm{~cm}$ x $20 \mathrm{~cm}$. Our pixel chamber is only $12 \mathrm{~cm} \mathrm{x} 12 \mathrm{~cm}$. The ground plane "sees" a lots of beam. The ground current fed through the signal plane and masks our signal display. But the result is encouraging enough that we decided to proceed to build a large chamber with more care in ground current return, which will not be a problem if our chamber is larger than the beam.

V. Pixel chamber:

We decided to use the spare Berkeley ion chamber housing for the pixel chamber. Fig.5 and 6 show the pixel plane and wire plane. 


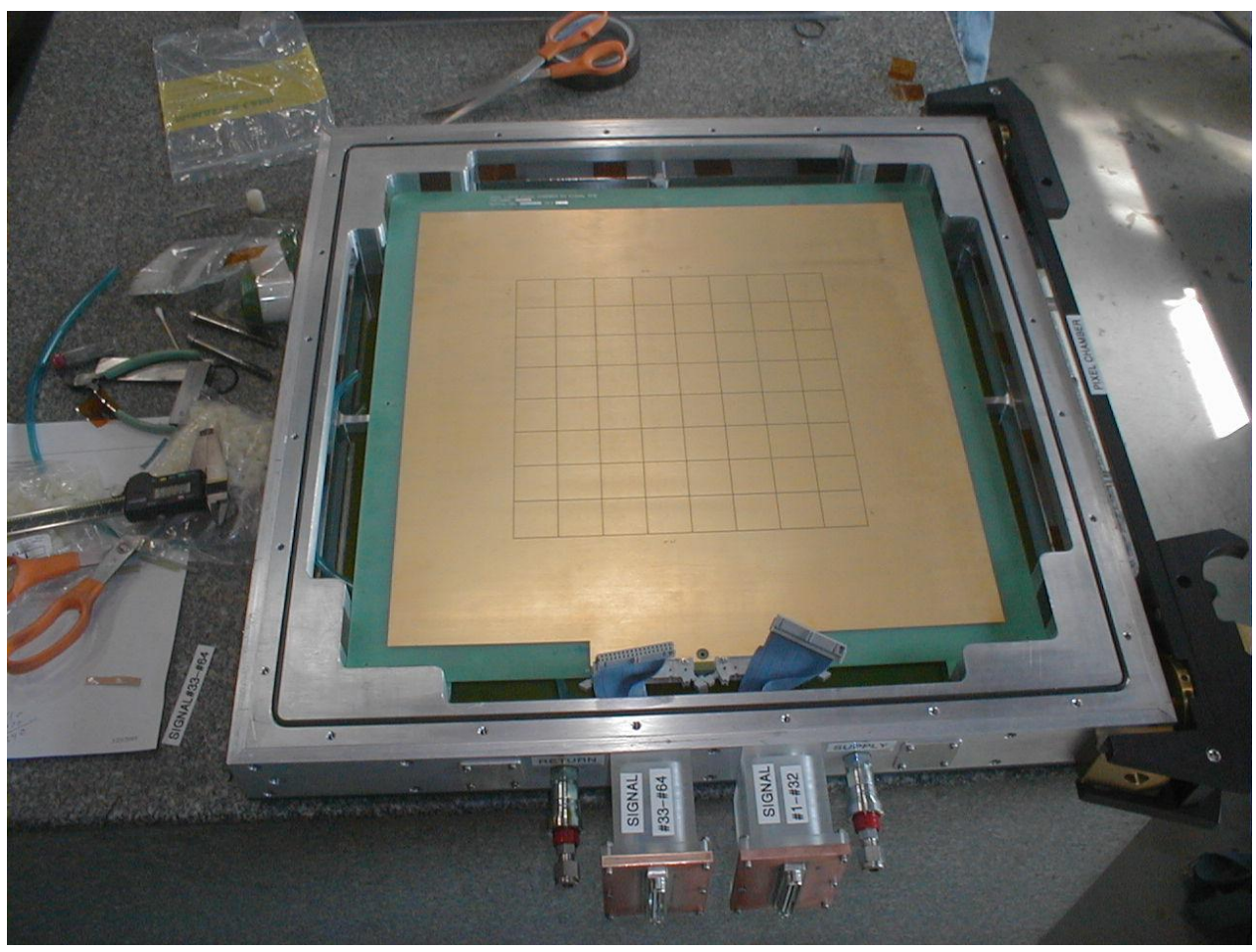

Fig. 5 Pixel Plane.

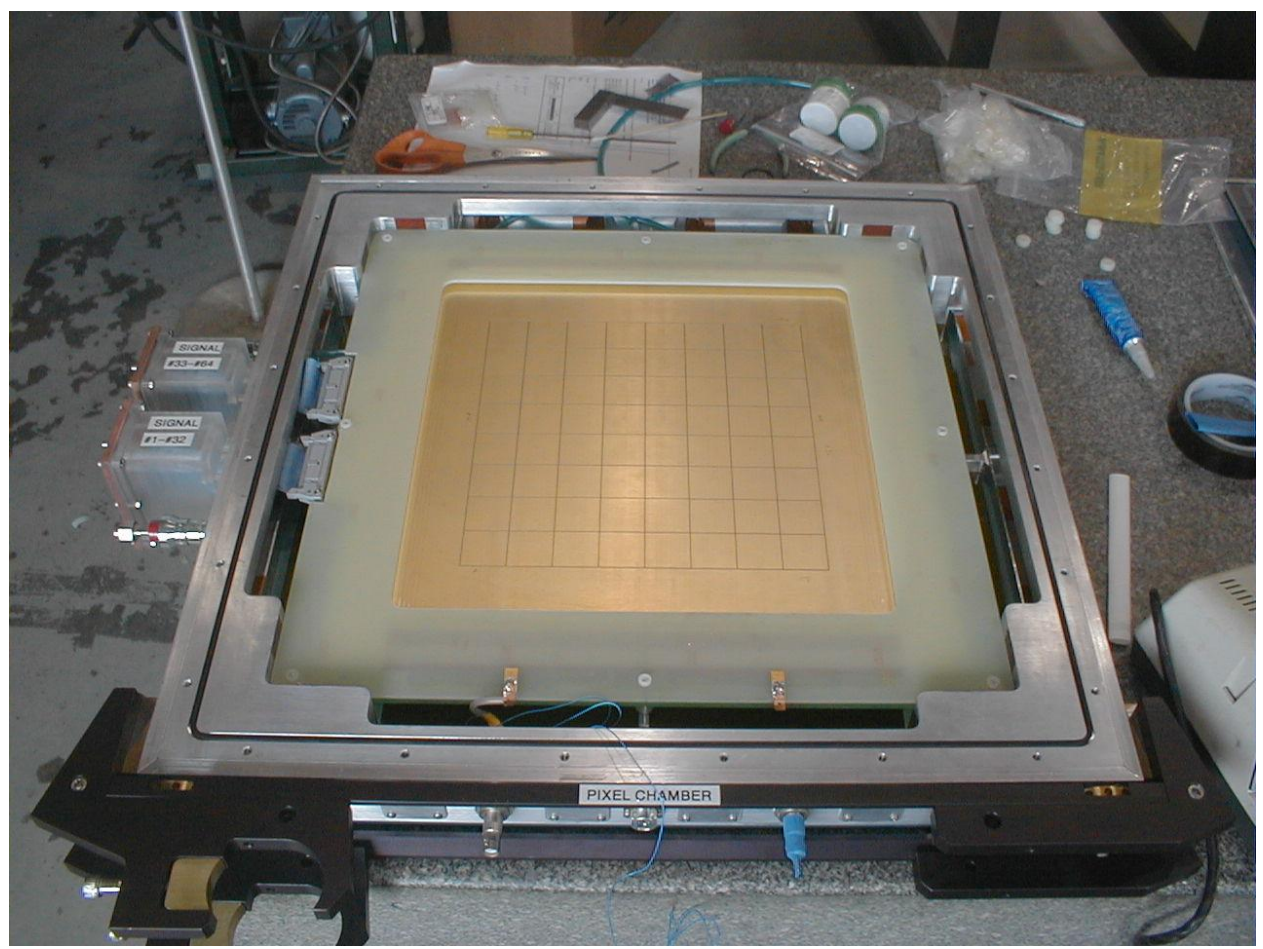

Fig. 6. Wire plane on top of Pixel Plane. 
The pixel size is $3 \mathrm{~cm} \times 3 \mathrm{~cm}$. We had $8 \times 8=64$ channels. The SWIC display has 64 channels, 32 horizontal and 32 vertical. The wire size is the same 18 micron, and wire spacing is $3 \mathrm{~mm}$. The $3 \mathrm{~mm}$ spacing is chosen so that each pixel faces exactly same number of wires. Note, the wire is only one dimensional and the pixel chamber is two dimensional. The uniformity of the wire size and spacing are very important to the uniformity of the chamber response. The gas we use for the chamber is Argon and $\mathrm{CO} 2$ with 50-50 mixture. We could change the ratio, 50-50, to get higher gain. Higher ratio allows us to run the chamber at higher voltage. The symptom of break down is "seeing" the integrator output increase without any beam. The initial try was limited by this effect. It did not occur to all the pixels but only a few. We conclude that there must be some dust/hair attached to the wire. This "dust" will start arcing and produce charge to be picked up by pixels. We try a few times to clean the chamber in a more "clean" environment. We did succeed to run the chamber up to 2400 volts. Fig. 7 shows the gain curve. The Blue curve is done with a Proton beam of $2 \times 10^{\wedge} 10$ Protons per pulse of the size $20 \times 20 \mathrm{~cm}$. The graph starts to curve at 1300 volts. This indicates the "saturation /recombination" effect started to affect the collection of ion/electron pairs. We then reduce the intensity by factor of about 10 and start with higher voltage, 1100 volts. The pink curve shows the graph. It was then renormalized to the Blue, proton curve at 1100 volts, producing the Yellow curve. The green curve was done with low intensity of small beam of Chorine, about 1000-2000 particles per pulse. It was then normalized to the yellow curve, at 1700 Volts. On the graph, the gain is close to 10000. In reality, the real gain is of factor 10 lower, or about. The initial increase, less than 5-600 volts, is due to increase in collection efficiency. It is important to 
see the curve, higher than 1200 volts, is almost pure exponential. We could use this to scale the data when we change the voltage. The fit is $y=.026 \times \operatorname{EXP}(.005 \mathrm{~V})$ where $\mathrm{V}$ is the Bias voltage. This factor, $.005 \times \mathrm{V}$, changed with wire and gap spacing. We did change the configuration later. The exponent should be re-measured for each chamber configuration.

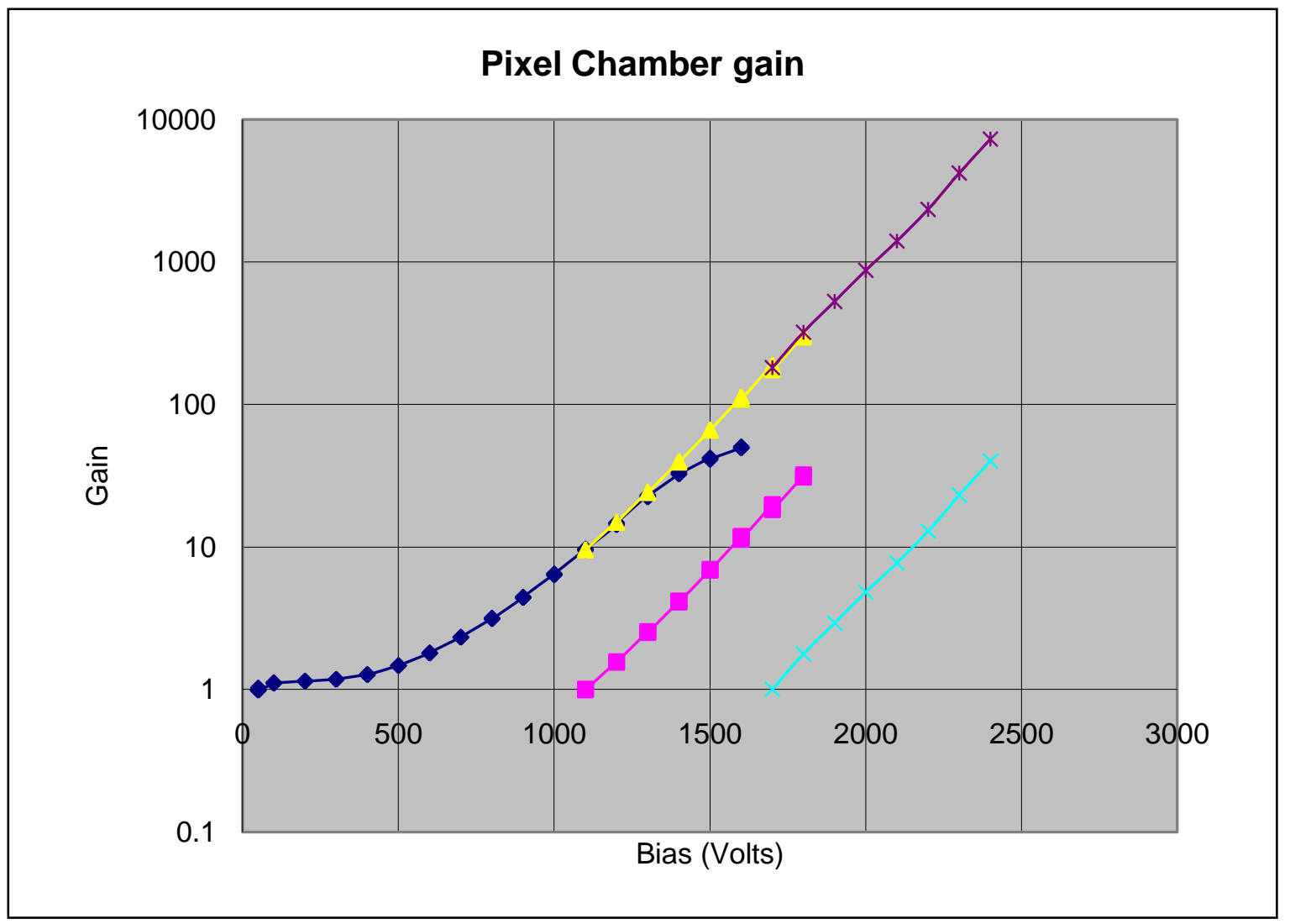

Fig. 7. Gain curve .

Blue Curve-Proton, $20 \times 20 \mathrm{~cm}$ at $2 \times 10^{\wedge} 10$ per pulse

Pink Curve--- Proton at 1/10 intensity

Yellow Curve-- Low intensity protron nromalized to High intensity proton.

Green Curve-Low intensity Chorine, 1-2000 particle per pulse, small beam Brown Curve-Green normalized to Yellow. 


\section{Display Program:}

Our normal SWIC display program was used to generate the above study. Fig. 8 shows the display. The display shows a repeat pattern of 8 . Since we had $8 \times 8$ pixel, the pattern repeat repeat 8 times. It is not very easy to visilized the display.

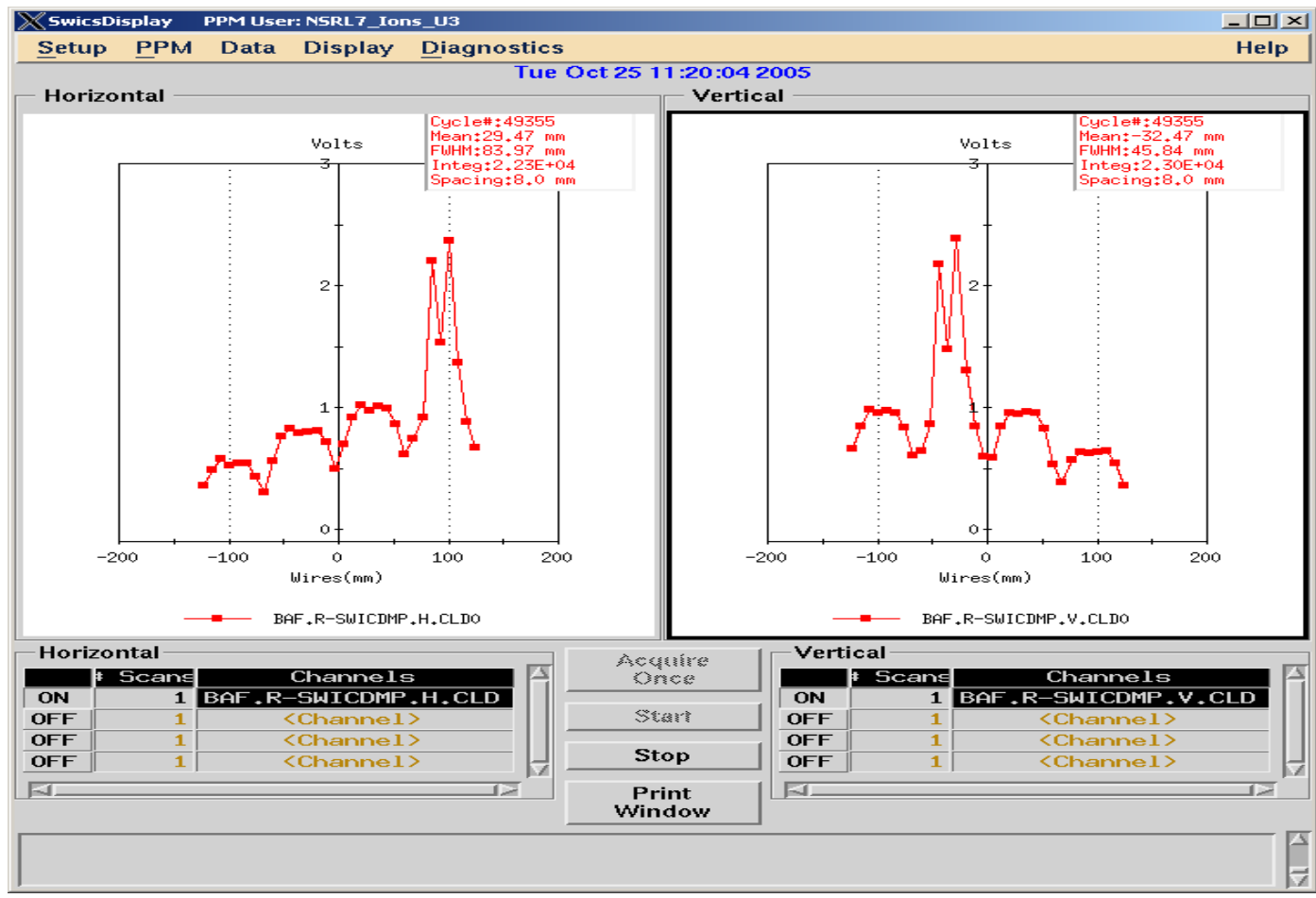

It was discovered that there is a good package of software available in the control system. It is J. G. Chart by Quest Software. The above pattern becomes. Fig 9. It shows a 4 beam spots. Fig. 10 shows a more normal operational beam distribtion. 


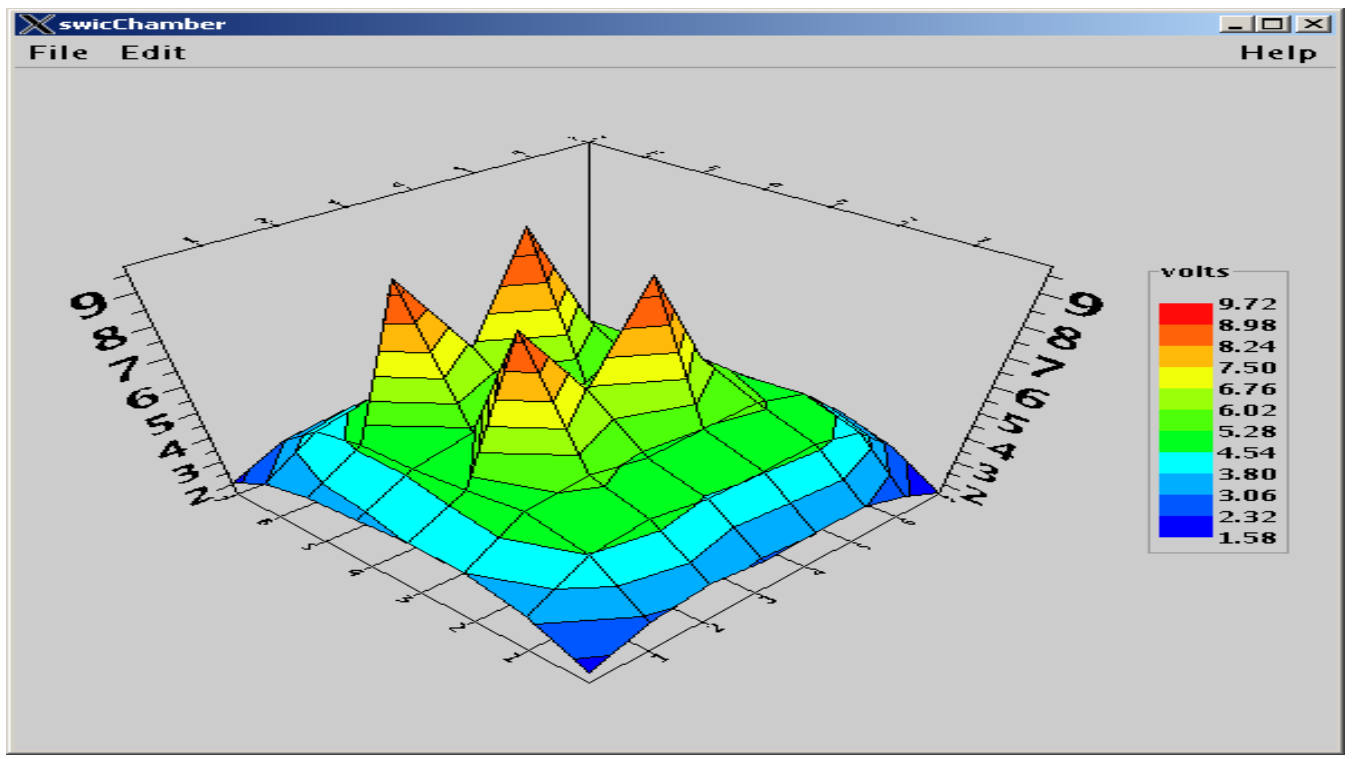

Fig.9, Image of beam after 4 holes collimator.

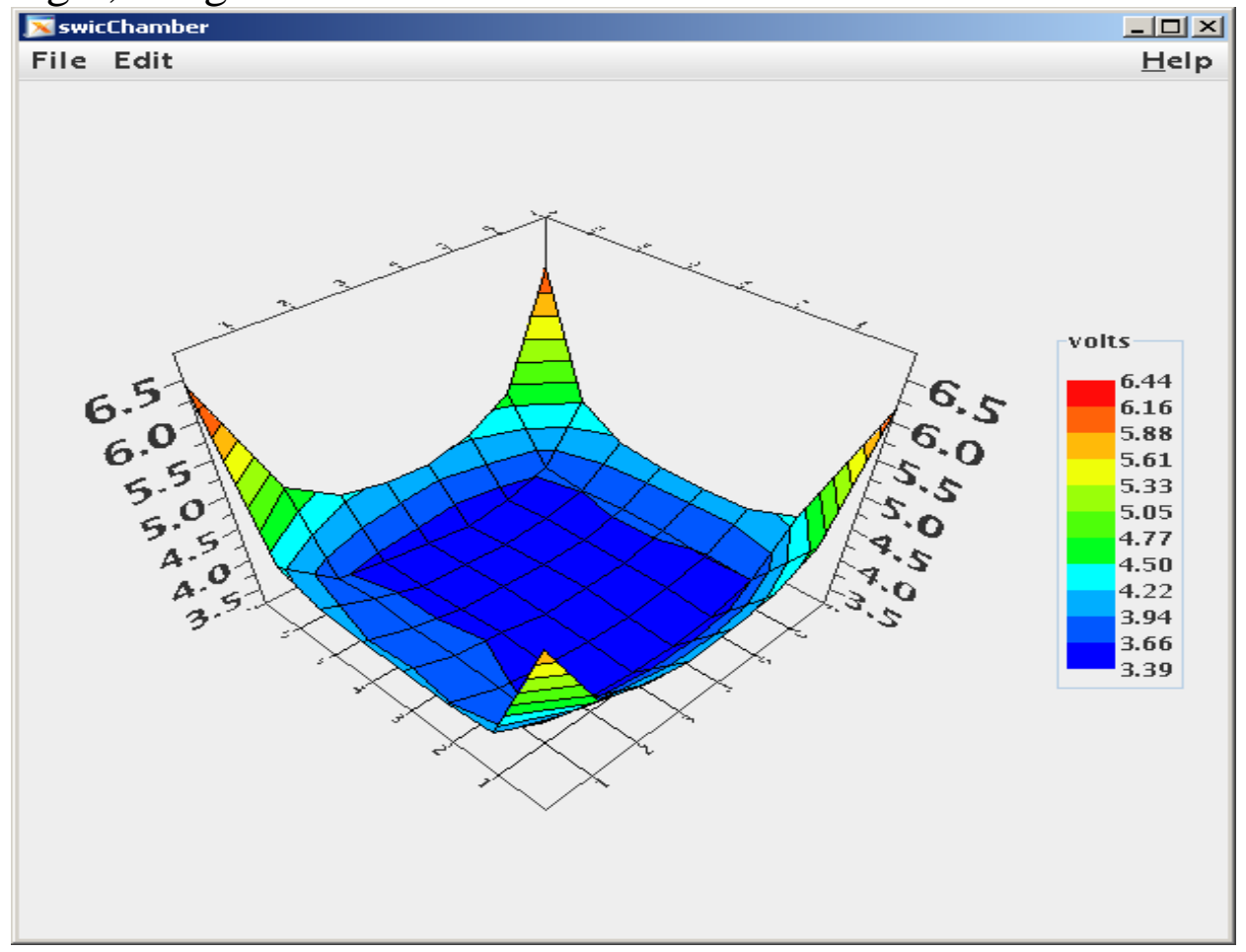

Fig. 10 Normal operational beam distribution.

This software allows us to change the display mode and rotate the projection. It is very useful and becomes more useful in low intensity operation. 
VII. Further application.

We are applying this technique, Ion chamber with gain, to other part of our operation. The most obvious area is low intensity dosimetry. We normally use Scintillator to account for low dose exposure. It is very desirable to connect the exposure with dose rather than counts. We are proceeding to make a thin pixel plan so we could move the chamber upstream in front of the samples. We are also developing a new recycling integrator for this purpose. Our normal recycling integrator cannot digitize the signal because it had the opposite polarity. We can then use this chamber to cut off the beam at the requested dose.

The recycling integrator will be an important element for the large beam application. We will move the small pixel chamber up stream to act as beam monitor/cut off chamber. There will be a larger pixel chamber built, 256 channels. That will be read out by recycling integrator. Before the electronics are done. We built a module to invert the signal so we could input the dosimetry chamber with gain into our system. The module works. It had slightly more noise than recycling integrator but we use it for low intensity dosimetry study. 